\title{
PRESERVATION OF PRIMARY FOREST CHARACTERISTICS DESPITE FRAGMENTATION AND ISOLATION IN A FOREST REMNANT FROM VIÇOSA, MG, BRAZIL ${ }^{1}$
}

\author{
Markus Gastauer², Marcos Eduardo Guerra Sobral ${ }^{3}$ e João Augusto Alves Meira-Neto ${ }^{4}$
}

\begin{abstract}
According to its owners, the Forest of Seu Nico (FSN) from the Viçosa municipality, Minas Gerais, Brazil, never has been logged and is therefore considered a primary forest. Nevertheless, the forest patch suffered impacts due to selective wood and non-timber extraction, fragmentation and isolation. Aim of this study was to test if the FSN, despite impacts, preserved characteristics of primary forests, which are elevated percentages of non-pioneer $(>90 \%)$, animal-dispersed ( $>80 \%)$, understory $(>50 \%)$ and endemic species $(\sim 40 \%)$. For that, all trees with diameter at breast height equal or major than $3.2 \mathrm{~cm}$ within a plot of 100 x 100 m were identified. With 218 tree species found within this hectare, the FSN's species richness is outstanding for the region. The percentages of non-pioneer ( $92 \%$ ), animal-dispersed ( $85 \%)$, understory (55\%) and endemic species $(39.2 \%)$ from the FSN fulfill the criteria proposed for primary forest. Therefore, we conclude that the FSN maintained its characteristics as a primary forest which highlights its importance for the conservation of biotic resources in the region, where similar fragments are lacking or not described yet.
\end{abstract}

Keywords: Endemism; Species composition; Species richness.

\section{PRESERVAÇÃO DAS CARACTERÍSTICAS DE FLORESTAS PRIMÁRIAS, APESAR DE FRAGMENTAÇÃO E ISOLAMENTO NUM REMANESCENTE FLORESTAL EM VIÇOSA, MG, BRASIL}

\begin{abstract}
RESUMO - Segundo seus proprietários, a Floresta de Seu Nico (FSN), situada no Município de Viçosa, Minas Gerais, Brasil, nunca sofreu corte raso, porém é considerada floresta primária. No entanto, o fragmento florestal sofreu impactos por extração seletiva de madeira e produtos não madeireiros, além de fragmentação e isolamento. O objetivo desse estudo foi testar se a FSN, apesar de impactos, preservou características de florestas primárias, que são porcentagens elevadas de espécies não pioneiras (>80\%), espécies dispersas por animais (>90\%), espécies de sub-bosque (> 50\%) e espécies endêmicas ( 40\%). Para isso, todas as árvores com diâmetro à altura do peito igual ou superior a 3,2 cm, dentro de uma parcela de $100 \times 100$ $m$, foram identificadas. Com 218 espécies de árvores encontradas dentro da parcela, a riqueza de espécies da FSN é elevada na região. As porcentagens de espécies não pioneiras (92\%), dispersas por animais (85\%), de sub-bosque (55\%) e endêmicas (39,2\%) da FSN cumprem os critérios propostos para florestas primárias. Porém, conclui-se que a FSN manteve suas características como floresta primária, que destaca a sua importância para a conservação dos recursos bióticos da região, onde fragmentos semelhantes estão faltando ou, ainda, não foram descritos.
\end{abstract}

Palavras-chave: Endemismo; Composição de espécies; Riqueza de espécies.

\footnotetext{
${ }^{1}$ Recebido em 10.10.2014 aceito para publicação em 29.10.2015.

${ }^{2}$ Universidade Federal de Viçosa, Programa de Pós-Graduação em Ecologia, Viçosa, MG - Brasil. E-mail: <mgasti@hotmail.com>.

${ }^{3}$ Universidade Federal de São João Del-Rei, Departamento de Ciências Naturais, São João Del Rei, MG - Brasil. E-mail: $<$ marcus_sobral@hotmail.com>.

${ }^{4}$ Universidade Federal de Viçosa, Centro de Ciências Biológicas e da Saúde, Departamento de Biologia Vegetal, Viçosa, MG

- Brasil. E-mail: <j.meira@ufv.br>.
} 


\section{INTRODUCTION}

Before the Europeans arrived in South America, the Atlantic Forest covered between 1.300.000 and $1.500 .000 \mathrm{~km}^{2}$ distributed nearly along the entire Brazilian coast. Today, this formerly continuous area is highly fragmented with only 11 to $16 \%$ of the original vegetation cover remaining; most of existing forests are secondary fragments that recover from some type of land clearance (RIBEIRO et al., 2009a). Primary forest remnants, i.e., forest patches never logged or clear-cut (VELOSO et al., 1991) that have experienced little or no human disturbance (GIBSON et al., 2011), are rare. Primary forests show higher species richness and diversity than secondary forests (BROWNING et al., 2010; STYRING et al., 2010), although they might have suffered selective wood or non-timber extraction. Furthermore, indirect impacts such as fragmentation and isolation create extinction debts (LIRA et al., 2012) initiating their degradation (GASTAUER; MEIRA-NETO, 2013).

But primary and secondary forests differ not only in species richness and diversity (PETERSON; CARSON, 2008 ) but also in regeneration, stratification and dispersion strategies of species forming these forests (NUNES et al., 2003). Because environmental conditions, such as nutrient supply and light availability in the understory, change gradually during succession (LAURANCE et al., 2002), the percentage of pioneer species is higher in the initial than in later stages of succession. Due to reduced light availability in primary, old-growth forests, the proportion of understory species should be higher within primary forests than secondary fragments (GUARIGUATA; OSTERTAG, 2001). Furthermore, wind-dispersed species dominate the species assemblage in the initial phases of succession (INGLE, 2003) and are substituted by animal-dispersed species in advanced stages. Finally, widespread species are expected to colonize disturbed or logged habitats faster than endemic species with a narrow geographic distribution (MORI et al., 1981).

The proportions of non-pioneer, animal-dispersed, understory and endemic species have been used to estimate the time necessary to regenerate the Atlantic Forests after a disturbance (LIEBSCH et al., 2008). Liebsch et al. (2008) postulated for primary remnants from the Atlantic Forests to hold a proportion of less than $10 \%$ of pioneer species, more than $50 \%$ of understory species, more than $80 \%$ of animal-dispersed species and around
$40 \%$ of species endemic to the Atlantic Forest biome. In this article, we use these characteristics to test if the Forest of Seu Nico (FSN), an unlogged forest patch that underwent only selective wood extraction and exploitation of further, non-timber products (CAMPOS et al., 2006), maintained its characteristics as a primary, old-growth forest despite fragmentation and isolation.

\section{MATERIALAND METHODS}

Located at $20^{\circ} 47^{\prime} \mathrm{S}$ and $42^{\circ} 50^{\prime} \mathrm{W}$ at the Bom Sucesso Farm, Viçosa, Minas Gerais State, Brazil, the FSN is a forest fragment covering approximately 36 ha of a small valley. According to the Köppen system, the climate of Viçosa is characterized as Cwb (PEEL et al., 2007). Cwb is a mesothermic climate with mild, rainy summers and dry winters. The predominant soils are oxisols, but inceptisols are found on the slopes and neosols are found in the sedimentation areas. According to Velloso et al. (1991), the predominant vegetation is characterized as a Submontane Seasonal Semideciduous Forest.

For the sample, the plot method has been applied (NEWTON, 2007): one hectare was divided in 100 quadratic plots of $10 \times 10 \mathrm{~m}$. All trees with diameter at breast height (dbh) larger than $3.2 \mathrm{~cm}$ were identified. Nomenclature and systematic classification of species follows the database of Missouri Botanical Garden (2013). All species have been classified according to their regeneration, stratification and dispersion guilds. Thus, two regeneration (or successional) guilds have been defined (SIMBERLOFF; DAYAN, 1991). The pioneer species require direct light for all phases of development, while non-pioneer species are able to develop one or more phases of their life in shady conditions of the understory (GANDOLFI et al., 1995). Classifying species in stratification guilds separates understory species reaching heights up to $15 \mathrm{~m}$ only from canopy species regularly exceeding this value (LIEBSCH et al., 2008). Furthermore, two dispersion guilds were distinguished (animal-dispersed species and other vectors).

Species classification in regeneration, and dispersion guilds was performed by consulting the following literature (sorted in alphabetic order, not by relevance): Appolinário et al. (2005), Aquino and Barbosa (2009), Araújo et al. (2005), Carvalho et al. (2007), Carvalho and Nascimento (2009), Lopes et al. (2002), Nunes et al. (2003), Oliveira-Filho et al. (2004, 
Table 1 - Geographic distribution and affiliation to ecological guilds of tree species from the one hectare plot in the FSN. 'yes' is species endemic to the Atlantic Rain Forest biome, 'no' is species not endemic to the Atlantic Rain Forest biome, 'pio' is pioneer species, 'npio' is non-pioneer species, 'zoo' is animal-dispersed species, 'nzoo' is not animaldispersed species, 'us' is understory species and 'cp' is canopy species.

Tabela 1 - Distribuição geográfica e afiliação a guildas ecológicos de espécies de árvores encontradas numa parcela de um hectare na FSN, isto é, espécies endêmicas do bioma da Mata Atlântica não o são não endêmicas do bioma da Mata Atlântica, 'pio'é espécie pioneira, 'npio'é espécie não pioneira, 'zoo'é espécie dispersa por animais, 'nzoo' é espécie não dispersa por animais, 'us'é espécie de sub-bosque e 'cp'é espécie do dossel.

\begin{tabular}{|c|c|c|c|}
\hline Family & Species name & $\begin{array}{l}\text { Geographic } \\
\text { distribution }\end{array}$ & $\begin{array}{l}\text { Affiliation to } \\
\text { ecological guilds }\end{array}$ \\
\hline Achariaceae & Carpotroche brasiliensis (Raddi) A. Gray & yes & npio-zoo-us \\
\hline \multirow[t]{4}{*}{ Anacardiaceae } & Astronium fraxinifolium Schott & no & npio-nzoo-us \\
\hline & Astronium graveolens Jacq. & no & npio-nzoo-cp \\
\hline & Tapirira guianensis Aubl. & no & npio-zoo-us \\
\hline & Tapirira obtusa (Benth.) J.D. Mitch. & no & npio-zoo-cp \\
\hline \multirow[t]{8}{*}{ Annonaceae } & Annona cacans Warm. & yes & npio-zoo-cp \\
\hline & Guatteria australis A. St.-Hil. & yes & npio-zoo-us \\
\hline & Guatteria villosissima Saint-Hilaire & no & npio-zoo-us \\
\hline & Guatteria sp. 1 & nc & nc-zoo-us \\
\hline & Guatteria sp. 2 & $\mathrm{nc}$ & nc-zoo-us \\
\hline & Rollinia laurifolia Schltdl & yes & npio-zoo-cp \\
\hline & Xylopia brasiliensis Spreng. & yes & npio-zoo-cp \\
\hline & Xylopia sericea A. St.-Hil. & no & pio-zoo-us \\
\hline \multirow[t]{4}{*}{ Apocynaceae } & Aspidosperma olivaceum Müll. Arg. & yes & npio-nzoo-us \\
\hline & Aspidosperma polyneuron Müll. Arg. & yes & npio-nzoo-us \\
\hline & Aspidosperma subincanum Mart. & no & npio-nzoo-us \\
\hline & Tabernaemontana hystrix Steud. & yes & pio-zoo-us \\
\hline Aquifoliaceae & Ilex cerasifolia Reissek & no & npio-zoo-us \\
\hline \multirow[t]{2}{*}{ Araliaceae } & Dendropanax cuneatus (DC.) Decne. \& Planch. & no & npio-zoo-us \\
\hline & Schefflera morototoni (Aub1.) Maguire et al. & no & pio-zoo-cp \\
\hline \multirow[t]{2}{*}{ Arecaceae } & Astrocaryum aculeatissimum (Schott) Burret & yes & npio-zoo-us \\
\hline & Euterpe edulis Mart. & no & npio-zoo-us \\
\hline Asteraceae & Vernonanthera diffusa (Less.) H.Rob. & no & pio-nzoo-cp \\
\hline \multirow[t]{3}{*}{ Bignoniaceae } & Jacaranda macrantha Cham. & no & npio-nzoo-cp \\
\hline & Sparattosperma leucanthum (Vell.) K. Schum. & no & npio-nzoo-us \\
\hline & Handroanthus chrysotrichus (Mart. ex A. DC.) Mattos & no & npio-nzoo-us \\
\hline Boraginaceae & Cordia sellowiana Cham. & no & npio-zoo-cp \\
\hline \multirow[t]{3}{*}{ Burseraceae } & Protium heptaphyllum (Aubl.) Marchand & no & npio-zoo-cp \\
\hline & Protium warmingianum Marchand & no & npio-zoo-cp \\
\hline & Trattinnickia ferruginea Kuhlm. & yes & nc-zoo-cp \\
\hline Cannbaceae & Celtis iguanaea (Jacq.) Sarg. & no & pio-zoo-us \\
\hline Cardiopteridaceae & Citronella paniculata (Mart.) R.A. Howard & yes & npio-zoo-us \\
\hline Caricaceae & Jacaratia cf. heptaphylla (Vell.) A. DC. & yes & npio-zoo-cp \\
\hline \multirow[t]{4}{*}{ Celastraceae } & Maytenus floribunda Reissek & no & npio-zoo-us \\
\hline & Maytenus robusta Reissek & no & npio-zoo-us \\
\hline & Maytenus salicifolia Reissek & no & npio-zoo-cp \\
\hline & Salacia elliptica (Mart. ex Schult.) G. Don & no & npio-zoo-us \\
\hline \multirow[t]{2}{*}{ Chrysobalanaceae } & Hirtella hebeclada Moric. ex DC. & yes & npio-zoo-cp \\
\hline & Licania belemii Prance & yes & nc-zoo-cp \\
\hline \multirow[t]{4}{*}{ Clusiaceae } & Garcinia brasiliensis Mart. & no & npio-zoo-us \\
\hline & Kielmeyera albopunctata Saddi & yes & nc-nc-us \\
\hline & Tovomita glazioviana Engl. & yes & npio-zoo-us \\
\hline & Tovomitopsis saldanhae Eng1. & yes & npio-zoo-cp \\
\hline Combretaceae & Terminalia glabrascens Mart. & no & npio-nzoo-us \\
\hline Elaeocarpaceae & Sloanea hirsuta (Schott) Planch ex Benth. & yes & npio-zoo-us \\
\hline
\end{tabular}


Table 1 - Cont

Tabela 1 - Cont.

\begin{tabular}{|c|c|c|c|}
\hline Family & Species name & $\begin{array}{l}\text { Seographic } \\
\text { listribution }\end{array}$ & $\begin{array}{l}\text { Affiliation to } \\
\text { ecological guilds }\end{array}$ \\
\hline \multirow[t]{2}{*}{ Erythroxylaceae } & Erythroxylum daphnites Mart. & no & nc-zoo-us \\
\hline & Erythroxylum pelleterianum A. St.-Hil. & no & npio-zoo-us \\
\hline \multirow[t]{11}{*}{ Euphorbiaceae } & Alchornea glandulosa Poepp. \& Endl. & no & pio-zoo-cp \\
\hline & Alchornea triplinervia (Spreng.) Müll. Arg. & no & npio-zoo-cp \\
\hline & Aparisthmium cordatum (A.Juss.) Baill. & no & npio-nzoo-us \\
\hline & Croton floribundus Spreng. & no & pio-nzoo-cp \\
\hline & Mabea fistulifera Mart. & no & pio-nzoo-cp \\
\hline & Maprounea guianensis Aubl. & no & npio-nzoo-cp \\
\hline & Pera glabrata (Schott) Poepp. ex Baill. & no & npio-zoo-cp \\
\hline & Sapium glandulosum (L.) Morong & no & pio-zoo-cp \\
\hline & Euphorbiaceae sp. 1 & nc & nc \\
\hline & Euphorbiaceae sp. 2 & nc & nc \\
\hline & Euphorbiaceae sp. 3 & $\mathrm{nc}$ & $\mathrm{nc}$ \\
\hline \multirow[t]{21}{*}{ Fabaceae } & Andira fraxinifolia Benth. & no & npio-zoo-us \\
\hline & Apuleia leiocarpa (Vogel) J.F. Macbr. & no & npio-nzoo-cp \\
\hline & Copaifera langsdorffii Desf. & no & npio-zoo-cp \\
\hline & Dalbergia nigra (Vell.) Allemao ex Benth. & yes & npio-nzoo-cp \\
\hline & Hymenaea sp. & $\mathrm{nc}$ & $\mathrm{nc}$ \\
\hline & Inga capitata Desv. & no & npio-zoo-cp \\
\hline & Inga cylindrica (Vell.) Mart. & no & npio-zoo-cp \\
\hline & Inga vera Willd. & no & npio-zoo-cp \\
\hline & Inga $\mathrm{sp}$. & $\mathrm{nc}$ & nc-zoo-cp \\
\hline & Lonchocarpus cultratus (Vell.) A.M.G. Azevedo \& H.C. Lima & ma no & npio-nzoo-cp \\
\hline & Machaerium caratinganum Kuhlm. \&Hoehne & yes & npio-nzoo-nc \\
\hline & Machaerium nyctitans (Vell.) Benth. & yes & npio-nzoo-cp \\
\hline & Machaerium sp. & nc & nc-nzoo-nc \\
\hline & Melanoxylon brauna Schott & yes & npio-nzoo-cp \\
\hline & Ormosia arborea (Vell.) Harms & yes & npio-zoo-cp \\
\hline & Peltophorum dubium (Spreng.) Taub. & no & npio-zoo-cp \\
\hline & Piptadenia gonoacantha (Mart.) J.F. Macbr. & no & pio-nzoo-cp \\
\hline & Pseudopiptadenia contorta (DC.) G.P. Lewis \& M.P. Lima & no & npio-nzoo-cp \\
\hline & Swartzia acutifolia Vogel & yes & npio-zoo-cp \\
\hline & Swartzia myrtifolia $\mathrm{Sm}$. & no & npio-zoo-us \\
\hline & Fabaceae sp. & $\mathrm{nc}$ & $\mathrm{nc}$ \\
\hline Humiriaceae & Vantanea obovata (Nees \& Mart.) Benth. & no & nc-zoo-us \\
\hline Lacistemaceae & Lacistema pubescens Mart. & no & npio-zoo-us \\
\hline \multirow[t]{15}{*}{ Lauraceae } & Aniba firmula (Nees \& C. Mart.) Mez & no & npio-zoo-cp \\
\hline & Cinnamomum glaziovii (Mez) Kosterm. & yes & npio-zoo-cp \\
\hline & Cryptocarya moschata Nees \& C. Mart. ex Nees & yes & npio-zoo-cp \\
\hline & Nectandra lanceolata Nees \& Mart. & no & npio-zoo-cp \\
\hline & Nectandra oppositifolia Nees \& Mart. & no & npio-zoo-cp \\
\hline & Ocotea corymbosa (Meisn.) Mez & no & npio-zoo-cp \\
\hline & Ocotea dispersa (Nees \& Mart.) Mez & yes & npio-zoo-us \\
\hline & Ocotea odorifera (Vell.) Rohwer & yes & npio-zoo-cp \\
\hline & Ocotea pulchella (Nees \& Mart.) Mez & no & npio-zoo-cp \\
\hline & Ocotea silvestris Vattimo-Gil & yes & npio-zoo-us \\
\hline & Ocotea sp. & nc & nc-zoo-us \\
\hline & Phyllostemonodaphne geminiflora (Mez) Kosterm. & yes & npio-zoo-us \\
\hline & Urbanodendron verrucosum (Nees) Mez & yes & npio-zoo-us \\
\hline & Lauraceae sp. 1 & nc & nc-zoo-us \\
\hline & Lauraceae sp. 2 & nc & nc-zoo-us \\
\hline
\end{tabular}

Revista Árvore, Viçosa-MG, v.39, n.6, p.985-994, 2015 
Table 1 - Cont.

Tabela 1 - Cont

\begin{tabular}{|c|c|c|c|}
\hline Family & Species name & $\begin{array}{l}\text { Seographic } \\
\text { listribution }\end{array}$ & $\begin{array}{l}\text { Affiliation to } \\
\text { ecological guilds }\end{array}$ \\
\hline \multirow[t]{2}{*}{ Lecythidaceae } & Cariniana estrellensis (Raddi) Kuntze & no & npio-nzoo-cp \\
\hline & Cariniana legalis (Mart.) Kuntze & yes & npio-nzoo-cp \\
\hline Lythraceae & Lafoensia glyptocarpa Koehne & no & npio-nc-cp \\
\hline \multirow[t]{4}{*}{ Malvaceae } & Ceiba speciosa (A. St.-Hil.) Ravenna & no & npio-nzoo-cp \\
\hline & Eriotheca candolleana (K. Schum.) A. Robyns & no & npio-nzoo-us \\
\hline & Luehea grandiflora Mart. & no & npio-nzoo-us \\
\hline & Sterculia curiosa (Vell.) Taroda & yes & npio-zoo-cp \\
\hline \multirow[t]{6}{*}{ Melastomataceae } & Miconia brunnea Mart. ex DC. & yes & nc-zoo-us \\
\hline & Miconia budlejoides Triana & yes & npio-zoo-us \\
\hline & Miconia cinnamomifolia (DC.) Naudin & yes & pio-zoo-us \\
\hline & Miconia minutiflora (Bonpl.) DC. & no & nc-zoo-cp \\
\hline & Miconia tristis Spring & yes & npio-zoo-us \\
\hline & Mouriri glazioviana Cogn. & no & npio-zoo-us \\
\hline \multirow[t]{10}{*}{ Meliaceae } & Cabralea canjerana (Vell.) Mart. & no & npio-zoo-cp \\
\hline & Cedrela fissilis Vell. & no & npio-zoo-cp \\
\hline & Guarea guidonia (L.) Sleumer & no & npio-zoo-cp \\
\hline & Guarea kunthiana A.Juss. & no & npio-zoo-cp \\
\hline & Guarea macrophylla Vahl & no & npio-zoo-cp \\
\hline & Guarea pendula R.da Silva Ramalho, A.L. Pinheiro \& T.D. Penn. & yes & npio-zoo-us \\
\hline & Trichilia catigua A. Juss. & no & npio-zoo-us \\
\hline & Trichilia emarginata (Turcz.) C. DC. & yes & npio-zoo-us \\
\hline & Trichilia lepidota Mart. & no & npio-zoo-cp \\
\hline & Trichilia pallida $\mathrm{Sw}$. & no & npio-zoo-cp \\
\hline Monimiaceae & Mollinedia schottiana (Spreng.) Perkins & no & npio-zoo-us \\
\hline \multirow[t]{10}{*}{ Moraceae } & Brosimum guianense (Aubl.) Huber & no & npio-zoo-cp \\
\hline & Clarisia ilicifolia (Spreng.) Lanj. \& Rossberg & no & npio-zoo-us \\
\hline & Ficus enormis (Mart. ex Miq.) Mart. & no & npio-zoo-cp \\
\hline & Ficus gomelleira Kunth \& C.D. Bouché & no & npio-zoo-cp \\
\hline & Ficus luschnathiana (Miq.) Miq. & no & npio-zoo-cp \\
\hline & Helicostylis tomentosa (Poepp. \& Endl.) Rusby & no & npio-zoo-cp \\
\hline & Maclura tinctoria (L.) D. Don ex Steud. & no & npio-zoo-cp \\
\hline & Naucleopsis oblongifolia (Kuhlm.) Carauta & yes & npio-zoo-cp \\
\hline & Sorocea bonplandii (Baill.) W.C. Burger, Lanj. \& Wess. Bo & oerno & npio-zoo-us \\
\hline & Sorocea hilariana (Cesar.) Bureau & yes & npio-zoo-us \\
\hline \multirow[t]{2}{*}{ Myristicaceae } & Virola bicuhyba (Schott ex. Spreng.) Warb. & yes & npio-zoo-cp \\
\hline & Virola gardneri (A. DC.) Warb. & yes & npio-zoo-cp \\
\hline \multirow[t]{19}{*}{ Myrtaceae } & Calyptranthes brasiliensis Spreng. & yes & npio-zoo-us \\
\hline & Campomanesia xanthocarpa (Mart.) O. Berg & yes & npio-zoo-us \\
\hline & Eugenia cf. lambertiana DC. & no & npio-zoo-us \\
\hline & Eugenia dodonaeifolia Cambess. & yes & npio-zoo-us \\
\hline & Eugenia florida DC. & no & npio-zoo-us \\
\hline & Eugenia leptoclada O.Berg & yes & npio-zoo-us \\
\hline & Marlierea excoriata Mart. & yes & npio-zoo-us \\
\hline & Marlierea suaveolens Cambess. & yes & npio-zoo-us \\
\hline & Marliereateuscheriana (O.Berg) D.Legrand & yes & npio-zoo-us \\
\hline & Myrcia anceps (Spreng.) O. Berg & yes & npio-zoo-us \\
\hline & Myrcia laxiflora Cambess. & yes & npio-zoo-us \\
\hline & Myrcia pubipetala Miq. & yes & npio-zoo-us \\
\hline & Myrcia splendens (Sw.) DC. & no & npio-zoo-us \\
\hline & Myrciaria floribunda (H.West ex Willd.) O.Berg & no & npio-zoo-us \\
\hline & Myrciaria pallida $\mathrm{O}$. Berg & yes & npio-zoo-us \\
\hline & Neomitranthes $\mathrm{sp}$. & nc & nc-zoo-us \\
\hline & Plinia cf. grandifolia (Mattos) Sobral & yes & npio-zoo-us \\
\hline & Psidium cf. oblongatum $\mathrm{O}$. Berg & yes & nc-zoo-us \\
\hline & Myrtaceae sp. & $\mathrm{nc}$ & nc-zoo-nc \\
\hline
\end{tabular}


Table 1 - Cont

Tabela 1 - Cont.

\begin{tabular}{|c|c|c|c|}
\hline Family & Species name & $\begin{array}{l}\text { Geographic } \\
\text { distribution }\end{array}$ & $\begin{array}{l}\text { Affiliation to } \\
\text { ecological guilds }\end{array}$ \\
\hline \multirow[t]{4}{*}{ Nyctaginaceae } & Guapira hirsuta (Choisy) Lundell & yes & npio-zoo-us \\
\hline & Guapira opposita (Vell.) Reitz & no & npio-zoo-cp \\
\hline & Pisonia ambigua Griseb. & no & npio-zoo-cp \\
\hline & Nyctaginaceae sp. & $\mathrm{nc}$ & nc-zoo-nc \\
\hline Ochnaceae & Ouratea polygyna Engl. & yes & npio-zoo-us \\
\hline \multirow{3}{*}{ Olacaceae } & Heisteria silvianii Schwacke & yes & npio-zoo-us \\
\hline & Tetrastylidium grandiflorum (Baill.) Sleumer & yes & npio-zoo-us \\
\hline & Oleaceae sp. & $\mathrm{nc}$ & nc-zoo-nc \\
\hline \multirow[t]{2}{*}{ Phyllanthaceae } & Hieronyma alchorneoides Allemão & no & npio-zoo-cp \\
\hline & Margaritaria nobilis L. f. & no & npio-zoo-cp \\
\hline \multirow{2}{*}{ Piperaceae } & Piper arboreum Aubl. & no & npio-zoo-us \\
\hline & Piper gigantifolium C. DC. & no & nc-zoo-us \\
\hline \multirow[t]{3}{*}{ Primulaceae } & Ardisia cf. catharinensis $\mathrm{Mez}$ & yes & nc-zoo-us \\
\hline & Cybianthus fuscus Mart. & no & nc-zoo-nc \\
\hline & Myrsine umbellata Mart. & yes & npio-zoo-us \\
\hline Rhamnaceae & Colubrina glandulosa Perkins & no & npio-zoo-cp \\
\hline Rosaceae & Prunus myrtifolia (L.) Urb. & no & npio-zoo-cp \\
\hline \multirow[t]{16}{*}{ Rubiaceae } & Alseis floribunda $\mathrm{Schott}$ & no & npio-zoo-us \\
\hline & Amaioua guianensis Aubl. & no & npio-zoo-us \\
\hline & Bathysa cuspidata (A.St. Hil.) Hook.f. ex K.Schum. & yes & npio-nzoo-us \\
\hline & Bathysa nicholsonii $\mathrm{K}$. Schum. & yes & npio-nzoo-us \\
\hline & Genipa americana $\mathrm{L}$. & no & npio-zoo-cp \\
\hline & Guettarda viburnoides Cham. \& Schltdl. & no & npio-zoo-us \\
\hline & Ixora gardneriana Benth. & yes & npio-zoo-us \\
\hline & Psychotria carthagenensis Jacq. & no & npio-zoo-us \\
\hline & Psychotria conjungens Müll. Arg. & yes & npio-zoo-us \\
\hline & Psychoria myriantha Müll. Arg. & yes & npio-zoo-us \\
\hline & Psychotria nuda (Cham. \& Schltdl.) Wawra & yes & npio-zoo-us \\
\hline & Psychotria vellosiana Benth. & no & npio-zoo-us \\
\hline & Psychotria sp. & $\mathrm{nc}$ & nc-zoo-nc \\
\hline & Randia ferox (Cham. \& Schltdl.) DC. & yes & npio-zoo-us \\
\hline & Rudgea jasminoides (Cham.) Müll.Arg. & no & npio-zoo-us \\
\hline & Rubiaceae sp. & $\mathrm{nc}$ & nc-zoo-nc \\
\hline \multirow[t]{2}{*}{ Rutaceae } & Zanthoxylum rhoifolium Lam. & no & npio-zoo-us \\
\hline & Hortia brasiliana Vand. ex DC. & yes & nc-zoo-cp \\
\hline Sabiaceae & Meliosma itatiaiae Urb. & yes & nc-zoo-us \\
\hline \multirow[t]{9}{*}{ Salicaceae } & Casearia arborea (Rich.) Urb. & no & npio-zoo-cp \\
\hline & Casearia decandra Jacq. & no & npio-zoo-cp \\
\hline & Casearia gossypiosperma Briq. & no & npio-nzoo-us \\
\hline & Casearia sylvestris $\mathrm{Sw}$. & no & pio-zoo-us \\
\hline & Casearia ulmifolia Vahl ex Vent. & no & npio-zoo-us \\
\hline & Macrothumia kuhlmannii (Sleumer) M.H.Alford & yes & nc-zoo-cp \\
\hline & Prockia crucis $\mathrm{P}$. Browne ex L. & no & npio-zoo-us \\
\hline & Xylosma prockia (Turcz.) Turcz. & no & npio-zoo-us \\
\hline & Salicaceae sp. & $\mathrm{nc}$ & nc-zoo-nc \\
\hline \multirow[t]{3}{*}{ Sapindaceae } & Allophylus edulis (A. St.-Hil. et al.) Hieron. ex Niederl. & no & npio-zoo-us \\
\hline & Cupania vernalis Cambess. & no & npio-zoo-cp \\
\hline & Matayba elaeagnoides Radlk. & no & npio-zoo-cp \\
\hline \multirow[t]{6}{*}{ Sapotaceae } & Chrysophyllum gonocarpum (Mart. \& Eichler ex Miq.) & Engl. no & npio-zoo-cp \\
\hline & Chrysophyllum lucentifolium Cronquist & no & npio-zoo-us \\
\hline & Chrysophyllum cf. marginatum (Hook. \& Arn.) Radlk. & no & npio-zoo-cp \\
\hline & Chrysophyllum sp. & $\mathrm{nc}$ & nc-zoo-nc \\
\hline & Pouteria caimito (Ruiz \& Pav.) Radlk. & no & npio-zoo-cp \\
\hline & Pradosia lactescens (Vell.) Radlk. & no & npio-zoo-cp \\
\hline
\end{tabular}

Revista Árvore, Viçosa-MG, v.39, n.6, p.985-994, 2015 


\begin{tabular}{|c|c|c|c|}
\hline Family & Species name & $\begin{array}{l}\text { Geographic } \\
\text { distribution }\end{array}$ & $\begin{array}{l}\text { Affiliation to } \\
\text { ecological guilds }\end{array}$ \\
\hline \multirow[t]{2}{*}{ Siparunaceae } & Siparuna guianensis Aubl. & no & npio-zoo-us \\
\hline & Siparuna reginae (Tul.) A. DC. & no & npio-zoo-us \\
\hline \multirow[t]{4}{*}{ Solanaceae } & Brunfelsia uniflora (Poh1) D. Don & no & npio-zoo-us \\
\hline & Cestrum mariqitense Kunth & no & nc-zoo-us \\
\hline & Cestrum sp. & nc & nc-zoo-nc \\
\hline & Solanaceae sp. & nc & nc-zoo-nc \\
\hline \multirow[t]{4}{*}{ Urticaceae } & Cecropia hololeuca Miq. & yes & pio-zoo-cp \\
\hline & Coussapoa floccosa Akkermans \& C.C. Berg & yes & nc-zoo-us \\
\hline & Coussapoa microcarpa (Schott) Rizzini & yes & npio-zoo-cp \\
\hline & Pourouma guianensis Aubl. & no & pio-zoo-cp \\
\hline Vochysiaceae & Qualea jundiahy Warm. & yes & npio-zoo-cp \\
\hline \multirow{2}{*}{ Unknown family } & Unidentified sp. 1 & nc & $\mathrm{nc}$ \\
\hline & Unidentified sp. 2 & nc & nc \\
\hline
\end{tabular}

2007), Paula et al. (2004) and Pinto et al. (2005). Information about species' stratification guild was retrieved from Oliveira-Filho (2014), geographic distribution was obtained from Stehmann et al. (2009) and Forzza et al. (2013); endemic species are those endemic to the Atlantic Forest biome. In the event of contradictory information for classification, the species were allocated to the guild indicated by the majority of references.

\section{RESULTS}

In the one hectare plot from the FSN, 2529 alive and 100 dead individuals with $\mathrm{dbh} \geq 3.2 \mathrm{~cm}$ were sampled. Altogether, 218 (morpho-)species belonging to139 genus and 52 families have been detected within the survey (Table 1). Due to lack of appropriate material (e.g. flowers) to provide a definite determination, 23 morphospecies were not identified until species level. Guild classification of these taxa was not always possible (Table 1).

From all classified species, only 14 species are recognized as pioneer species, so that $92 \%$ of all classified taxa are non-pioneer species. 179 or $85 \%$ of all species are dispersed by animals and 109 species or $55 \%$ of all 199 classified species are understory species. $39.2 \%$ of completely identified species are endemic to the Atlantic Rain Forest, while 118 or little more than 60 $\%$ occur in further biomes as well.

\section{DISCUSSION}

The FSN supports higher tree species richness than comparable plots from secondary forest in its neighborhood (RIBEIRO et al., 2009b; GASTAUER;
MEIRA-NETO, 2013). Furthermore, the species lists gathered in a one hectare plot within the FSN fulfills all criteria proposed by Liebsch et al. (2008) for oldgrowth, primary forests as there are less than $10 \%$ of non-pioneer, more than $80 \%$ of zoochorus, more than $50 \%$ understory species and about $40 \%$ of species endemic to the Atlantic Forest biome.

The Atlantic Forest is a heterogeneous ecosystem comprising more than 27 latitudinal degrees, with precipitation regimes ranging from arid conditions to more than 3,000 $\mathrm{mm}$ per year and altitudes from sea level up to $3000 \mathrm{~m}$ (STEHMANN et al., 2009). Therefore, the characteristics and their scores proposed by Liebsch et al. (2008) for primary evergreen forests from the Southern Atlantic Forest cannot be generalized for the entire Atlantic Forest and its associated ecosystems (ALMEIDA-NETO et al., 2008). Consequently, real scores for primary forests in the study region are unknown. Furthermore, the classification of a single species with all of its genotypic and phenotypic variability within a single guild might be subjective or inaccurate, although species classification is based on non-ambiguous definitions. Additionally, insufficient knowledge about species' ecology might influence guild classification. Therefore, alterations in the classification and the definition of maximal scores might change the results and influence the interpretation and conclusions.

Despite the above mentioned restrictions, we can conclude from the species list from the one hectare plot that the FSN has maintained its characteristics

Revista Árvore, Viçosa-MG, v.39, n.6, p.985-994, 2015 
as an old-growth, primary forest despite fragmentation and isolation, at least in some parts. In other parts, edge effects due to isolation or landscape fragmentation and the selective wood extraction that occurred within the fragment might have altered the primary forest characteristics or may alter them in the future, as indicated by a slight loss of species richness and diversity during the last decade (GASTAUER; MEIRA-NETO, 2013).

\section{CONCLUSION}

As the FSN is the only known forest fragment in the Viçosa municipality that has never been logged. Therefore, we expect that the FSN is the only forest patch that fulfills the criteria to be identified as a primary forest. Unique species richness, diversity and fulfillment of primary forest characteristics in a highly degraded and fragmented landscape emphasize the FSN's special role as a species pool for surrounding forest fragments and illustrate its insubstitutability for biological conservation.

\section{REFERENCES}

ALMEIDA-NETO, M.; CAMPASSI, F.; GALETTI, M.; JORDANO, P.; OLIVEIRA-FILHO, A.

Vertebrate dispersal syndromes along the Atlantic forest, broad-scale patterns and macroecological correlates. Global Ecology and

Biogeography, v. 17, n. 4, p.503-513, 2008.

APPOLINÁRIO, V.; OLIVEIRA FILHO, A.T.; GUILHERME, F.A.G. Tree population and community dynamics in a Brazilian tropical semideciduous forest. Revista Brasileira de Botânica, v.28, n.2, p.347-360, 2005.

AQUINO, C.; BARBOSA, L.M. Classes sucessionais e syndromes de dispersão de espécies arbóreas e arbustivas existents em vegetação ciliar remanescente (Conchal, SP), como subsídio para avaliar o potencial do fragmento como fonte de propágulos para enriquecimento de areas revegetadas no Rio Mogi-Guaçu, SP. Revista Árvore, v.33, n.2, p.349-358, 2009.

ARAÚJO, F.S.; MARTINS, S.V.; MEIRA NETO, J.A.A.; LANI, J.L.; PIRES, I.E. Florística da vegetação arbustivo-arbórea colonizadora de uma área degradada por mineração de caulim, em Brás Pires, MG. Revista Árvore, v. 29, n.6, p. 983992, 2005..3

Revista Árvore, Viçosa-MG, v.39, n.6, p.985-994, 2015
BROWNING, B.J.; JORDAN, G.J.; DALTON, P.J.; GROVE, S.J.; WARDLAW, T.J.; TURNER, P.A.M. Succession of mosses, liverworts and ferns on coarse woody debris, in relation to forest age and log decay in Tasmanian wet eucalypt forest.

Forest Ecology and Management, v.260, n.10, p.1896-1905, 2010.

CAMPOS, E.P.; SILVA, A.F.; MEIRA NETO, J.A.A.; MARTINS, S.V. Floristica e estrutura horizontal da vegetação arbórea de uma ravina em um fragmento florestal no município de Viçosa, MG. Revista Árvore, v.30, n.6, p.1045-1054, 2006.

CARVALHO, F.A.; NASCIMENTO, M.T.; BRAGA, J.M.A. Estrutura e composição florística do estrato arbóreo de um remanescente de Mata Atlântica submontana no município de Rio Bonito, RJ, Brasil (Mata Rio Vermelho). Revista Árvore, v.31, n.4, p.717-730, 2007.

CARVALHO, F.A.; NASCIMENTO, M.T. Estrutura diamétrica da comunidade e das principais populações arbóreas de um remanescente de Floresta Atlântica Submontana (Silva Jardim-RJ, Brasil). Revista Árvore, v.33, n.2, p.327-337, 2009.

FORZZA, R.C. (Coord.). Lista de Espécies da Flora do Brasil. Acessado em: julho 2013, Disponível em:http,//floradobrasil.jbrj.gov.br/

GANDOLFI, S.; LEITÃO FILHO, H.F.; BEZERRA, C.L.F. Levantamento florístico e caráter sucessional das espécies arbustivo-arbóreas de uma floresta mesófila semidecídua no município de Guarulhos, SP. Revista Brasileia de Biologia, v.55, n.4, p.753-767, 1995.

GASTAUER, M.; MEIRA-NETO, J.A.A. Community dynamics in a species-rich old-growth forest patch from Viçosa, Minas Gerais, Southeastern Brazil. Acta Botanica Brasilica, v.27, n.2, p.270-285, 2013.

GUARIGUATA, M.R.; OSTERTAG, R. Neotropical secondary forest succession: changes in structural and functional characteristics. Forest Ecology and Management, v.148, n.3, p.185-206, 2001. 
GIBSON, L. Primary forests are irreplaceable for sustaining tropical biodiversity. Nature, v.478, p.378-381, 2011.

INGLE, N.R. Seed dispersal by wind, birds, and bats between Philippine montane rainforest and sucessional vegetation. Oecologia, v.134, p.251$261,2003$.

LAURENCE, W.F.; LOVEJOY, T.E.; VASCONCELOS, H.L.; BRUNA, E.M.; DIDHAM, R.K.; STOUFFER, P. C.; GASCON, C.; BIERREGAARD, R.O.; LAURANCE, S.G.; SAMPAIO, E. Ecosystem decay of Amazonia forest fragments: a 22-year investigation. Conservation Biology, v.16, n.3, p.605-618, 2002.

LIEBSCH, D.; MARQUES, M.C.M.; GOLDENBERG, R. How long does the Atlantic Rain Forest take to recover after a disturbance? Changes in species composition and ecological features during secondary succession. Biological Conservation, v.141, p.1717-1725, 2008.

LIRA, P.K.; EWERS, R.M.; BANKS-LEITE, C.; PARDINI, R.; METZGER, J.P. Evaluating the legacy of landscape history: extinction debt and species credit in bird and small mammal assembalges in the Brazilian Atlantic Forest. Journal of Applied Ecology, v.49, p.1325$1333,2012$.

LOPES, W.P.; ALESSANDRO DE PAULA, A.; SEVILHA, A.C.; SILVA, A.F. Composição da flora arbórea de um trecho de Floresta Estacional no Jardim Botânico da Universidade Federal de Viçosa (Face Sudoeste), Viçosa, Minas Gerais. Revista Árvore, v.26, n.3, p.339-347, 2002.

\section{MISSOURI BOTANICAL GARDEN.}

Tropicos.org. Acessed: 12 Dec. 2013. Available at: http://www.tropicos.org.

MORI, S.A.; BOOM, B.M.; PRANCE, G.T. Distribution patterns and conservation of eastern Brazilian coastal forest species. Brittonia, v.33, n.3, p.233-245, 1981.

NEWTON, A.C. Forest ecology and conservation: a handbook of techniques. Oxford: Oxford University Press, 2007.

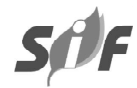

NUNES, Y.R.F.; MENDONÇA, A.V.R.; OLIVEIRAFILHO, A.T.; BOTEZELLI, L.; MACHADO, E.L.M.Variações da fisionomia, diversidade e composição de guildas da comunidade arbórea em um fragmento de floresta semidecidual em Lavras, MG. Acta Botanica Brasilica, v. 17, n.2, p.213-229, 2003.

OLIVEIRA-FILHO, A.T.. NeoTropTree, Flora arbórea da Região Neotropical: Um banco de dados envolvendo biogeografia, diversidade e conservação. Belo Horizonte: Universidade Federal de Minas Gerais, 2014. Acessado em: 7 out. 2014. Disponível em: http:// www.icb.ufmg.br/treeatlan/

OLIVEIRA-FILHO, A.T.; Carvalho,D.A.; Vilela, E.A.; Curi, N.; Fontes, M.A.L. Diversity and structure of the tree community of a fragment of tropical secondary forest of the Brazilian Atlantic Forest domain 15 and 40 years after logging. Revista Brasileira de Botânica, v.27, n.4, p.685-701, 2004

OLIVEIRA-FILHO, A.T.; CARVALHO, W.A.C.; MACHADO, E.L.M.; HIGUCHI, P.; APPOLINÁRIO, V.; CASTRO, G.C.; SILVA, A.C.; SANTOS, R.M.; BORGES, L.F.; CORRÊA, B.S.; ALVES, J.M. Dinâmica da comunidade e populações arbóreas da borda e interior de um remanescente florestal na Serra da Mantiqueira, Minas Gerais, em um intervalo de cinco anos (1999-2004). Revista Brasileira de Botânica, v.30, n.1, p.149-161, 2007

PAULA, A.; SILVA, A.F.; MARCO JÚNIOR, P.; SANTOS, F.A.M.; SOUZA, A.L. Sucessão ecológica da vegetação arbóres em uma Floresta Estacional Semidecidual, Viçosa, MG, Brasil. Acta Botanica Brasilica, v. 18, n.3, p.407-423, 2004.

PEEL, M.C.; FINLAYSON, B.L.; MCMAHON, T.A. Updated world map of the Köppen-Geiger climate classification. Hydrology and Earth System Sciences, v.11, p.1633-1644, 2007.

PETERSON, CJ.; CARSON, W.P. Process contraining woody species succession on abandoned pastures in the tropics: on the relevance of temperate models of succession. In: CARSON, P.C.; SCHNITZER, A.S. (Eds.).

Tropical forest community ecology. Singapore: Wiley-Blackwell, 2008. p.367-383.

Revista Árvore, Viçosa-MG, v.39, n.6, p.985-994, 2015 
PINTO, L.V.A. et al. Estudo da vegetação como subsídio para propostas de recuperação das nascentes da Bacia hidrográfica do Riberão Santa Cruz, Lavras, MG. Revista Árvore, v.29, n.5, p.775-793, 2005.

RIBEIRO, M.C.; METZGER, J.P.; MARTENSEN, A.C.; PONZONI, F.J.; HIROTA, M.M. The Brazilian Atlantic Forest: How much is left, and how is the remaining forest distributed? Implications for conservation. Biological Conservation, v. 142, n.6, p.1141-1153, 2009a.

RIBEIRO, S.C.; JACOVINE, L.A.G.; SOARES, C.P.B.; MARTINS, S.V.; SOUZA, A.L.; NARDELLI, A.M.B. Quantificação de biomassa e estimativa de estoque de carbono em uma floresta madura no município de Viçosa, Minas Gerais. Revista Árvore, v.33, n.5, p.917-926, 2009b.

SIMBERLOFF, D.; DAYAN, T. The guild concept and the structure of ecological communities.

\author{
Annual Review of Ecology, Evolution, \\ and Systematics, v.22, p.115-143, 1991. \\ STEHMANN, J.R.; FORZZA, R.C.; SALINO, A.; \\ SOBRAL, M.G.; COSTA, D.P.; KAMINO, L.H.Y. \\ (Org.). Plantas da Floresta Atlântica. Rio \\ de Janeiro: Instituto de Pesquisas Jardim \\ Botânico, 2009. \\ STYRING, A.R.; RAGAI, R.; UNGGANG, J.; \\ STUEBING, R.; HOSNER, P.A.; SHELDON, F.H. \\ Bird community assembly in Bornean industrial \\ tree plantations: Effects of forest age and \\ structure. Forest Ecology and \\ Management, v.261, n.3, p.531-544, 2011. \\ VELOSO, H.P.; RANGEL FILHO, A.L.R.; LIMA, \\ J.C.A. Classificação da vegetação \\ brasileira, adaptada a um sistema \\ universal. Rio de Janeiro: IBGE, Departamento \\ de Recursos Naturais e Estudos Ambientais, \\ 1991.
}

